\title{
Solutions of Poisson Equation by using Galerkin Method
}

\author{
Md. Anisur Rahman
}

\author{
Associate Professor, Department of Mathematics, Islamic University, Kushtia-7003, Bangladesh
}

\begin{abstract}
Numerical analysis is the area of mathematics and computer science that creates, analyzes, and implements algorithms for solving numerically the problems of continuous mathematics. The objective of this paper is to present a simple and accurate method to solve differential equations. To analyse Galerkin method with unknown functions as basic functions to solve Poisson equations numerically. This study compared the approximate solutions using various number of basis function with the exact solutions. The solution and graphical representation have been verified by Mathemetica and MATLAB. The results are shown in graphically. This paper is to understand the using of Poisson's equation in numerical analysis and the accuracy of the calculation of Galerkin's method.
\end{abstract}

Keywards: Function, Numerical, Galerkin method, Poisson equation, Differential equation

\section{INTRODUCTION}

Problems occur throughout the natural sciences, social sciences, medicine, engineering, and business. Beginning in the 1940 's, the growth in power and availability of digital computers has led to an increasing use of realistic mathematical models in science, medicine, engineering, and business; and numerical analysis of increasing sophistication has been needed to solve these more accurate and complex mathematical models of the world. Numerical analysis naturally finds applications in all fields of engineering and the physical sciences, but in the 21 st century also the life sciences and even the arts have adopted elements of scientific computations. Ordinary differential equations appear in celestial mechanics (planets, stars and galaxies); numerical linear algebra is important for data analysis; stochastic differential equations and Markov chains are essential in simulating living cells for medicine and biology. The field of numerical analysis predates the invention of modern computers by many centuries. Linear interpolation was already in use more than 2000 years ago. Many great mathematicians of the past were preoccupied by numerical analysis, as is obvious from the names of important algorithms like Newton's method, Lagrange interpolation polynomial, Gaussian elimination, or Euler's method [1].

Numerical analysis is the study of algorithms that use numerical approximation for the problems of mathematical analysis [2]. In other words, numerical analysis is the area of mathematics and computer science that creates, analyzes, and implements algorithms for solving numerically the problems of continuous mathematics. Such problems originate generally from real-world applications of algebra, geometry, and calculus, and they involve variables which vary continuously. Numerical methods are mathematical methods that give approximate solutions to mathematical problems based on arithmetic operations involving numbers with finite number of digits. It gives only approximations to the correct or exact mathematical solution. Galerkin method forms a class of numerical methods for solving differential equations. In [3] extended the original discontinuous Galerkin method to linear convection diffusion equations. In [4] extended discontinuous Galerkin methods further to compressible Navier-Stokes equations. In [5] work was generalized to become the well known local discontinuous Galerkin methods through work by [6]. Otherwise, in the discontinuous Galerkin community, several formulations have been proposed to solve PDEs involving second-order spatial derivatives (Manzanero et al., 2018). The last three decades, advances in the use of computational methods in rock mechanics have been impressive especially in specific numerical methods [7]. The analytical methods are very useful in geo-mechanics because they provide results with very limited effort and highlight the most important variables that determine the solution of a problem [8]. Analyzed the simplest, and most common, stochastic model for population processes, including those from biochemistry and cell biology, are continuous time Markov chains [9]. Define Convection-diffusion equations model a variety of physical phenomena [10]. Presents a numerical method based on the Mac Cormack finite difference scheme is presented [11]. The method was developed for simulating two-dimensional overland flow with spatially variable infiltration and micro topography using the hydrodynamic flow equations. In the mathematical field of Numerical analysis, interpolation is a method of constructing new data points within the range of a discrete set of known data points. Many different interpolation methods exist. In this review, this is mainly based on three main categories: deterministic, probabilistic and other methods. Deterministic methods create a continuous surface by only using the geometric characteristics of point observations.

This study has an exploration of the significance of numerical analysis and provides better understanding Poisson equation. Galerkin refers to general finite element methods for partial differential equations in which differential 


\section{International Advanced Research Journal in Science, Engineering and Technology}

Vol. 6, Issue 12, December 2019

operators are approximated by weak forms through the usual integration by parts. In particular, Galerkin methods allow the use of discontinuous finite element functions in the algorithm design. This paper is to understand the using of Poisson's equation in Numerical Analysis and the accuracy of the calculation of Galerkin's method.

\section{POISSON EQUATION}

A partial differential equation which is satisfied by the potential of a mass distribution inside domains occupied by the masses creating this potential .Poisson's equation is a basic example of a non-homogeneous equation of elliptic type. The equation was first considered by S. Poisson (1812). Poisson's equation is $\Delta \varphi=f$

where $\Delta$ is the Laplace operator, and $f$ and $\varphi$ are real or complex valued functions on a manifold. Usually, $f$ is given and $\varphi$ is sought. When the manifold is Euclidean space, the Laplace operator is often denoted as $\nabla^{2}$ and so Poisson's equation is frequently written as $\nabla^{2} \varphi=f$

The two dimensional Poisson equation has the following form:

In the Cartesian coordinate system, $\frac{\partial^{2} w}{\partial x^{2}}+\frac{\partial^{2} w}{\partial y^{2}}+\Phi(x, y)=0$

In the polar coordinate system, $\frac{1}{r} \frac{\partial}{\partial r}\left(r \frac{\partial w}{\partial r}\right)+\frac{1}{r^{2}} \frac{\partial^{2} w}{\partial \varphi^{2}}+\Phi(r, \varphi)=0$

In three dimensional Cartesian coordinates, it takes the form

$$
\left(\frac{\partial^{2}}{\partial x^{2}}+\frac{\partial^{2}}{\partial y^{2}}+\frac{\partial^{2}}{\partial z^{2}}\right) \phi(x, y, z)=f(x, y, z) \text {, when } f=0
$$

We retrieve Laplace's equation, Poisson's equation may be solved using a Green's function; a general exposition of the Green's function for Poisson's equation is given in the article on the screened Poisson equation.

\section{GALERKIN METHOD}

Galerkin Method, invented by Russian mathematician Boris Grigoryevich Galerkin. To apply this method, the variation form of the partial differential equation is obtained and tested with each of the basis as test-functions. Our objective is to numerically solve Poisson equation by choosing basis functions, then approximating the actual solution $u$ by a linear combination of this function $\tilde{u}=\sum_{j=1}^{N} c_{j} \phi_{j}$. Among all choices for the coefficients, we insist that the approximation satisfies.

\section{GALERKIN'S REQUIREMENT}

The residual $R=L(\mathrm{u})$ must be orthogonal to the basis elements used in the approximation, i.e., $\left(\phi_{i}, R\right)=c_{j}\left(\phi_{i}, L \phi_{j}\right)-\left(\phi_{i}, f\right)=0$

The basis functions must be independent satisfy the homogeneous boundary conditions of the problem and be dense in a space containing the actual solution.

Consider the vector space of real functions whose domain is the closed interval $[\mathrm{a}, \mathrm{b}]$. We define the inner product of two functions $f(x)$ and $g(x)$ as follows

$$
<f, g>=\int_{a}^{b} f(\mathrm{x}) \mathrm{g}(\mathrm{x}) \mathrm{dx}
$$

\section{MATHEMATICA FUNCTION}

To compute the inner product of two real functions over $[\mathrm{a}, \mathrm{b}],<f, g>=\int_{a}^{b} f(\mathrm{x}) \mathrm{g}(\mathrm{x}) \mathrm{dx}$ 


\section{International Advanced Research Journal in Science, Engineering and Technology}

Vol. 6, Issue 12, December 2019

\section{GALERKIN DIMENSION REDUCTION}

Choose a subspace $V_{n} \subset V$ of dimension $n$ and solve the projected problem:

Find $u_{n} \in V_{n}$ such that for all $v_{n} \in V_{n}, a\left(u_{n}, v_{n}\right)=f\left(v_{n}\right)$

Reducing the problem to a finite-dimensional vector subspace allows us to numerically compute $u_{n}$ as a finite linear combination of the basis vectors in $V_{n}$.

\section{GALERKIN ORTHOGONALITY}

The key property of the Galerkin approach is that the error is orthogonal to the chosen subspaces. Since $v_{n} \subset V$, we can use $v_{n}$ as a test vector in the original equation. Subtracting the two, we get the Galerkin orthogonality relation for the error, $\epsilon_{n}=u-u_{n}$ which is the error between the solution of the original problem $u$ and the solution of the Galerkin equation,

$u_{n} a\left(\in_{n}, v_{n}\right)=a\left(u, v_{n}\right)-a\left(u_{n}, v_{n}\right)=f\left(v_{n}\right)-f\left(v_{n}\right)=0$

\section{MATRIX FORM}

Since the aim of Galerkin's method is the production of a linear system of equations, we build its matrix form, which can be used to compute the solution by a computer program.

Let $e_{1}, e_{2}, \ldots \ldots \ldots, e_{n}$ be a basis for $V_{n}$. Then, it is sufficient to use these in turn for testing the Galerkin equation, i.e., find $u_{n} \in V_{n}$ such that $a\left(u_{n}, e_{i}\right)=f\left(e_{i}\right), \quad i=1,2,3, \ldots \ldots \ldots, n$. We expand $u_{n}$ with respect to this basis, $\tilde{u}=\sum_{j=1}^{N} c_{j} \phi_{j}$ and insert it into the equation above, to obtain $a\left(\sum_{j=1}^{n} u_{j} e_{j}, e_{i}\right)=u_{j} a\left(e_{j}, e_{i}\right)=f\left(e_{i}\right), i=1,2,3, \ldots \ldots \ldots, n$.

This previous equation is actually a linear system of equations $A_{u}=f$, where $A_{i j}=a\left(e_{j}, e_{i}\right), f_{i}=f\left(e_{i}\right)$

\section{ADVANTAGES OF COMPUTATIONAL GALERKIN METHOD}

The modern counterparts of traditional Galerkin method have evolve two quite separate directions, although both have sought to achieve maximum computational efficiency and trial functions have been almost exclusively polynomials. Early attempts to introduce a combined polynomials-Fourier-series solution were rather cumbersome. For piecewise linear polynomials as test and trial functions the resulting algebraic equations often coincide, for first and second derivatives in one dimension, with corresponding finite- difference expression. Each test function to a geometric location, linear independence of the resulting algebraic equation is assured. The traditional Galerkin method, are global methods, that the test and trial functions span the whole domain. As a result, comparable accuracy for a given number of unknowns is to be expected. However, by using orthogonal functions as a test and trial functions, the test functions are by definition linearly independent. The spectral methods works best when dealing with domains whose boundaries coincide with coordinate lines.

\section{DISADVANTAGES OF COMPUTATIONAL GALERKIN METHOD}

Like any other numerical methods, the Galerkin method has drawback also. The blind use this method would certainly results in a very inefficient algorithm. In comparison with finite elements using continuous basis functions, the number of variables is larger for an identical number of elements. 


\section{International Advanced Research Journal in Science, Engineering and Technology}

Vol. 6, Issue 12, December 2019

\section{SOLUTION OF POISSION EQUATION}

Consider the two dimensional Poisson's equation is given by

$\frac{\partial^{2} u}{\partial x^{2}}+\frac{\partial^{2} u}{\partial y^{2}}=k ; 0 \leq x, y \leq 1$

With $\mathrm{u}=0$ on the boundary $\mathrm{C}$ of the region $\mathrm{S}$. The functional for the above problem is given by,

$$
I(v)=\iint_{s} v\left(2 k-\frac{\partial^{2} v}{\partial x^{2}}-\frac{\partial^{2} v}{\partial y^{2}}\right) d x d y
$$

(2), where y vanishes on the boundary C.

Let $v(x, y)=\alpha x y(x-1)(y-1)$

Be a first approximation to u. Clearly, v satisfies the boundary conditions. i.e. $v=0$ on the boundary $\mathrm{C}$. The derivatives are given by

$$
\begin{array}{ll}
\frac{\partial v}{\partial x}=\alpha y(y-1)(2 x-1) ; & \frac{\partial v}{\partial y}=\alpha x(x-1)(2 y-1) \\
\frac{\partial^{2} v}{\partial x^{2}}=2 \alpha y(y-1) ; & \frac{\partial^{2} v}{\partial y^{2}}=2 \alpha x(x-1)
\end{array}
$$

Substituting for $\mathrm{v}$ in equation (2), We obtain

$I(v)=\int_{0}^{1} \int_{0}^{1} \alpha x y(x-1)(y-1)[2 k-2 \alpha y(y-1)-2 \alpha x(x-1)] d x d y$

Let

$$
\begin{aligned}
& a=\int_{0}^{1} \int_{0}^{1} x y(x-1)(y-1) d x d y=\frac{1}{36} \\
& b=\int_{0}^{1} \int_{0}^{1} x y^{2}(x-1)(y-1)^{2} d x d y=-\frac{1}{180} \\
& c=\int_{0}^{1} \int_{0}^{1} x^{2} y(x-1)^{2}(y-1) d x d y=-\frac{1}{180}
\end{aligned}
$$

Equation (5) now simplifies to $I(v)=2 k \alpha a-2 a^{2} b-2 a^{2} c$

Hence $\frac{\partial I}{\partial \alpha}=0=2 k a-4 a b-4 \alpha c$

Thus $\quad \alpha=\frac{a k}{2(b+c)}=-\frac{5}{4} k \quad$ [by using (6) ]

It follows that the required approximation for $\mathrm{u}$ is given by $u \approx v=-\frac{5}{4} k x y(x-1)(y-1)$

\section{APPROXIMATION}

Let a Poisson equation, $\frac{d^{2} u}{d x^{2}}+\frac{d^{2} u}{d y^{2}}=-2 \pi \sin (\pi x) \cos (\pi y)$

and the boundary conditions are given by, 


\section{International Advanced Research Journal in Science, Engineering and Technology}

Vol. 6, Issue 12, December 2019

$(\mathrm{x}, 0)=\sin (\pi \mathrm{x}),(\mathrm{x}, 1)=-\sin (\pi \mathrm{x}),(0, \mathrm{y})=0,(1, \mathrm{y})=0$

Equation (8) is to be discredited using a 2nd-order finite differences scheme and then solved over (9) different domains:

Case-1: $(\mathrm{x}, \mathrm{y}) \in[0,1] \times[0,1]$

and Case-2: $(x, y) \in[-2,2] \times[-2,2]$

It should be noted that the boundary conditions given above are for case- 1 and for case-2, it should be updated considering the exact solution. Due to the fact that the function $u$ is dependent on two variables, the discretization results in two separate indices which need to be converted into a single index using an appropriate method. Then the problem can be represented in matrix-form as $[\mathrm{L}]\{\mathrm{u}\}=\{\mathrm{f}\}$ and subsequently be solved by an algorithm. The results are to be plotted and compared to the exact solution of the partial differential equation given in the assignment as:

$u=\sin (\pi x) \cos (\pi y)$ (10)

The graph is the solution of Poisson equation using Galerkin methods are given below

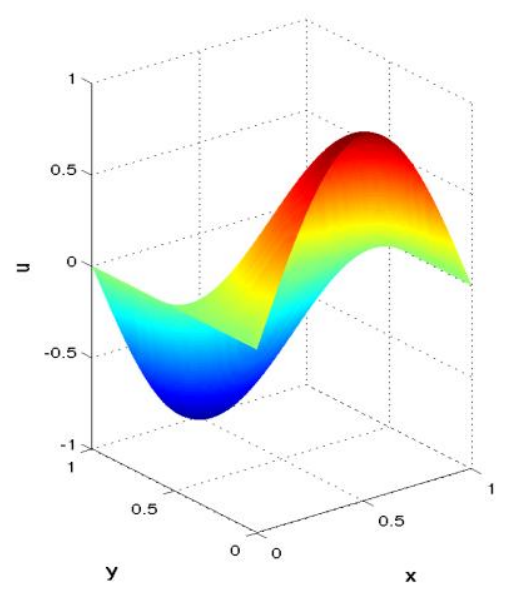

Figure 1: Graph of equation (10)

The solution of Poisson equation shows in the figure

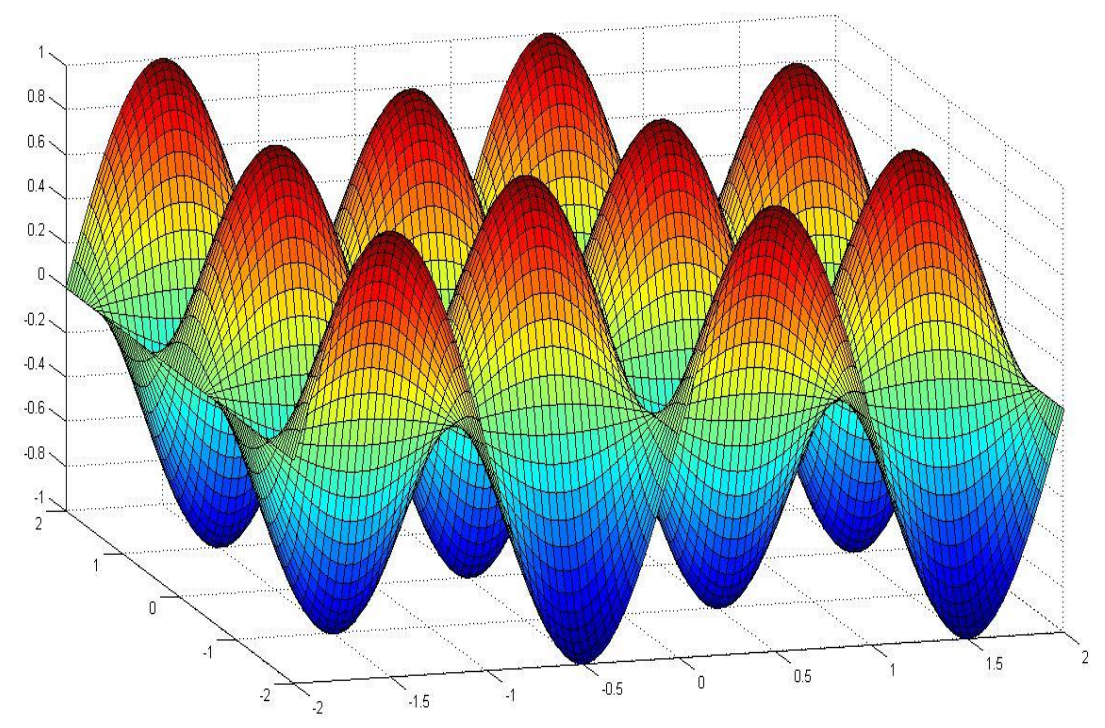

Figure 2: Graph of equation (8) 


\title{
International Advanced Research Journal in Science, Engineering and Technology
}

\author{
Vol. 6, Issue 12, December 2019
}

\section{DISCUSSION}

In this study to analyzed Galerkin method with unknown functions as basic functions to solve Poisson equations numerically. The main objective of this paper is to present a simple and accurate method to solve differential equations. The numerical results obtained by the proposed method are in good agreement with the exact solutions available in the literature. Otherwise compared the approximate solutions using various number of basis function with the exact solutions. The solution and graphical representation have been verified with Mathemetica and MATLAB. The results have showed graphically. It may realize that this method may be applied to solve numerically integral and differential equation.

\section{REFERENCES}

[1]. Leader, Jeffery J. (2004). "Numerical Analysis and Scientific Computation”, Addison Wesley, ISBN 0-201-73499-0.

[2]. Hildebrand, F. B. (1974). "Introduction to Numerical Analysis (2nd edition ed.)", McGraw-Hill. ISBN 0-07-028761-9.

[3]. Richter, R. G. ( 1992). "The discontinuous Galerkin method with diffusion". Math. Comp., Vol.58, No.198, P. 631-643,

[4]. Bassi F. and S. Rebay (1997). "A high-order accurate discontinuous finite element method for the numerical solution of the compressible NavierStokes equations", Journal of Computational Physics. Vol. 131, P. 267-279.

[5]. Manzanero J., A. M. Rueda-Ramírez, G. Rubio and E. Ferrer (2018), "The Bassi Rebay 1 scheme is a special case of the Symmetric Interior Penalty formulation for discontinuous Galerkin discretisations with Gauss-Lobatto points", Journal of Computational Physics, Vol. 363 , P.1-10.

[6]. Burn, B. C. and Chi-Wang Shu (1991). "The Runge-Kutta local projection Discontinuous Galerkin finite element method for scalar conservation laws", RAIRO Model. Math. Anal. Numer., Vol.25, No.03, P. 337-361.

[7]. Jing L. and J. A. Hudson (2002). "Numerical methods in rock mechanics”, International Journal of Rock Mechanics \& Mining Sciences, Vol.39, P. 409-427.

[8]. Bobet A. (2010). "Numerical Methods in Geomechanics", The Arabian Journal for Science and Engineering, Vol. 35, No. 1B.

[9]. Anderson, D. F. and M. Koyama (2012). "Weak Error Analysis of Numerical Methods for Stochastic Models of Population Processes", Department of Mathematics, University of Wisconsin, Madison, WI 53706.

[10]. Chertock, A. and A. Kurganov (2002). "On Splitting-Based Numerical Methods for Convection-Diffusion Equations", Department of Mathematics, North Carolina State University, Raleigh, NC 27695.

[11]. Fiedler, F. R. and J. A. Ramirez (2000). "A numerical method for simulating discontinuous shallow flow over an infiltrating surface", International Journal for Numerical Methods in Fluids, Vol. 32, P.219-240. 